\title{
Performance of the ATLAS Muon Spectrometer and Muon Identification
}

\author{
M.J. Woudstra*, for the ATLAS Collaboration \\ University of Massachusetts, Amherst, MA, USA \\ E-mail: martin.woudstra@cern.ch
}

\begin{abstract}
The large cosmic data samples collected in 2008 and 2009 by the ATLAS experiment have been used to study the performance of the muon spectrometer. Studies of the muon spectrometer performance in terms of calibration, alignment, tracking efficiency and momentum resolution are presented and provide an update with respect to results recently published. The results are also compared with a recently improved cosmic data simulation. Differences between data and simulation are discussed.

The performance of the ATLAS muon identification was studied with an integrated luminosity of $17 \mathrm{nb}^{-1}$ of LHC proton-proton collision data at a centre of mass energy of $7 \mathrm{TeV}$. Exploiting the redundancy in the muon identification at detector and reconstruction level, the performance of the identification steps could be checked with data. A brief mention is made on the 'prompt' muon fraction, the scale and resolution of the momentum measurement, and the rejection of the low momentum background from pion and kaon decays in flight.
\end{abstract}

35th International Conference of High Energy Physics

July 22-28, 2010

Paris, France

${ }^{*}$ Speaker. 


\section{Introduction}

In the ATLAS detector [1], four kinds of muons are distinguished depending on the way they are reconstructed:

Stand-alone muon: The muon trajectory is only reconstructed in the muon spectrometer, and is extrapolated to the beam line while correcting for the energy loss in the calorimeter.

Combined muon: The measurement of the stand-alone muon is combined with the measurement in the inner detector.

Segment tagged muon: A track in the inner detector is identified as a muon if its extrapolation to the muon spectrometer can be matched to (a) track segment(s) in the muon chambers.

Calorimeter tagged muon: A track in the inner detector is identified as a muon if the associated energy deposition in the calorimeters is compatible with a minimum ionizing particle.

\section{Results from Cosmics}

Hundreds of millions of cosmic ray events have been collected by the ATLAS experiment in the commissioning period in 2008 and 2009. The results on efficiency and resolution presented here use a sub-set of $21 \mathrm{M}$ events taken in fall 2009 , where the magnetic field in the muon spectrometer is at nominal strength. In addition, the tracks are required to be 'collision-like', i.e. they pass close to the p-p interaction point, which significantly reduces the statistics. As the cosmic muons mostly come in vertically, the performance could only be determined for the barrel region of the spectrometer.

The results presented here are updates to results published in [2], where details of these and other studies using the cosmics data can be found.

\subsection{Muon Spectrometer Calibration and Alignment}

At high momentum, the transverse momentum resolution of stand-alone muons is dominated by the calibration of the muon precision chambers and the internal alignment of the muon spectrometer.

The precision muon drift chambers (MDTs) measure the drift time which is then converted into a drift distance using the conversion function, which is determined from data in a calibration procedure. Each drift channel has a constant time offset (' $t_{0}$ ') for which the measured time has to be corrected to obtain the drift time. The $t_{0}$ 's are determined from data, but due to a lack of statistics these are not yet available for each individual channel separately. As a result, the obtained tracking precision is $20-50 \%$ worse than what has been achieved in the test-beam.

Runs taken without magnetic field in the muon spectrometer are used to check the chamber alignment by looking at the sagitta of the tracks, which is zero on average for the straight tracks. The overall average sagitta is within $40 \mu \mathrm{m}$ in the end-caps, while in the barrel typical average values are $30 \mu \mathrm{m}$ for the large sectors and $70 \mu \mathrm{m}$ for the small sectors.

\subsection{Efficiency from Cosmics}

The efficiency of the stand-alone muons is determined by selecting events with high quality inner detector tracks which are going through the barrel of the muon spectrometer. Then it is checked 
if a muon stand-alone track is reconstructed in top hemisphere and/or in the bottom hemisphere. The average efficiencies are $(97.4 \pm 0.1) \%$ and $(95.2 \pm 0.1) \%$ for the top and bottom hemispheres respectively. These numbers are higher than the ones published in [2] due to an improved alignment of the muon chambers and due to refinements in the muon reconstruction software. The lower efficiency in the bottom hemisphere is due to the uninstrumented regions occupied by the support structures of the ATLAS detector.

\subsection{Momentum resolution from Cosmics}

The transverse momentum resolution of the muon spectrometer was determined by comparing the two halves (i.e. from the top and bottom hemispheres) of the same muon track. Cuts are applied on the angles differences between the two track halves to ensure they belong to the same track. Figure 1 shows the resolution of the stand-alone muons as a function of the transverse momentum for data and simulation, for the small sectors of the muon spectrometer barrel.

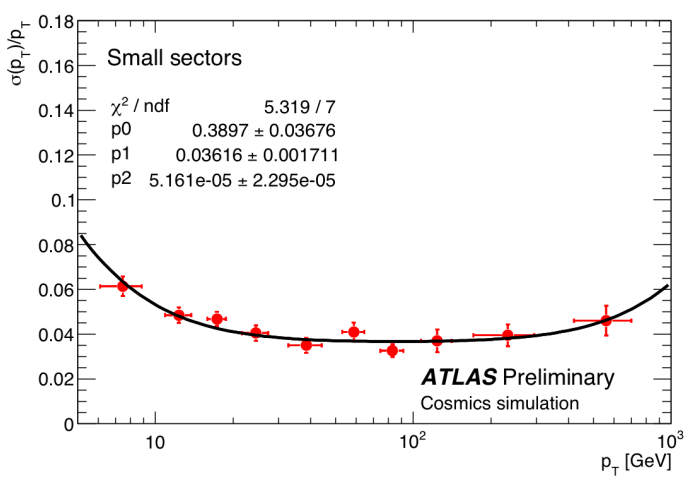

(a)

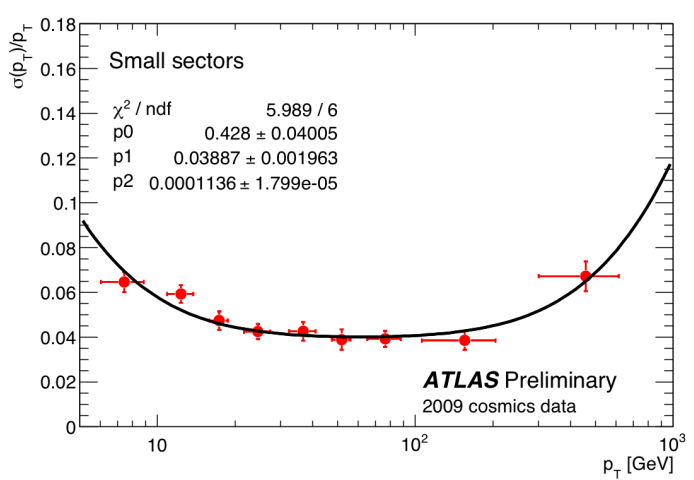

(b)

Figure 1: Resolution of the transverse momentum of the stand-alone muons in the small sectors of the barrel for simulation (a) and data (b).

The resolution function shown in figure 1 is $\sigma_{p_{T}} / p_{T}=p_{0} / p_{T} \oplus p_{1} \oplus p_{2} \cdot p_{T}$, and the fitted values of the parameters for the small sectors are given in the figure. For the large sectors the numbers are given in table 1.

Table 1: Fitted parameter values of the transverse momentum resolution function for large sectors

\begin{tabular}{r|c|c|c} 
Parameter & $p_{0}[\mathrm{GeV}]$ & $p_{1}[-]$ & $p_{2}\left[\mathrm{GeV}^{-1}\right]$ \\
\hline simulation & $0.29 \pm 0.02$ & $0.0297 \pm 0.0013$ & $(0.186 \pm 0.016) \cdot 10^{-3}$ \\
data & $0.31 \pm 0.03$ & $0.0388 \pm 0.0014$ & $(0.248 \pm 0.018) \cdot 10^{-3}$
\end{tabular}

The $p_{0}$ parameter, which models the energy loss fluctuations in the Calorimeter, is compatible between data and simulation, which means that the Calorimeter material description in the simulation is realistic. The $p_{1}$ parameter, which models the multiple scattering in the muon spectrometer, is worse in data than in the simulation, especially in the large sectors, which points to missing material in the simulation of the muon spectrometer. The $p_{2}$ parameter, which models the intrinsic spectrometer resolution, is worse in data than in simulation, which indicates that the alignment and calibration in the detector are not yet optimal. 


\section{Results from $7 \mathrm{TeV} p-p$ collisions}

The results corresponding to a detailed study with the first $17 \mathrm{nb}^{-1}$ of integrated luminosity of $p-p$ collisions at a center of mass energy of $7 \mathrm{TeV}$ are available in [3] and [4]. Here we briefly mention some of those studies.

The double momentum measurement for the combined muons in ATLAS, in the inner detector and in the muon spectrometer, is used to distinguish 'prompt' muons (mainly from decays of $b$ and $c$ quarks) from (late) decays in flight of $\pi$ 's and $K$ 's. As a discriminating variable the relative momentum imbalance of the muon spectrometer (MS) compared to the inner detector (ID) is used: $\Delta p / p_{I D}$ where $\Delta p=p_{I D}-p_{M S}-p_{C A L O}$ and $p_{C A L O}$ is the parametrized average energy loss in the calorimeter, which was determined from Monte Carlo studies.

To determine the 'prompt' muon fraction in data, the distribution of $\Delta p / p_{I D}$ for 'prompt' muons, as well as for $\pi$ and $K$ decays, is obtained from Monte Carlo simulation. The 'prompt' muon fraction increases, as expected, with increasing momentum. For example, in the rapidity range $1.8<|\eta|<2.5$ the fraction of $\pi$ and $K$ decays reduces from $\sim 50 \%$ in the range $4<p_{T}<5 \mathrm{GeV}$ to $\sim 4 \%$ in the range $10<p_{T}<20 \mathrm{GeV}$.

In studying the relative momentum scale and resolution between the inner detector and the muon spectrometer, the separation of the decays in flight is essential to avoid a bias in the results. In this case, the distribution of $\Delta p / p_{I D}$ for the 'prompt' muons is modeled with a function: a convolution of a Gaussian (to model the resolution) and a Landau (to model the energy loss in the calorimeter). The relative momentum scale and resolution are then obtained from this function.

To test the background rejection of $\pi$ and $K$ decays of the ATLAS muon identification, a pure sample of $K_{S}^{0} \rightarrow \pi^{+} \pi^{-}$decay candidates is selected, where the highest $p_{T}$ track of the pair of inner detector tracks is used as a probe. In a sample of $\sim 600 \mathrm{k}$ probes, $(0.070 \pm 0.012) \%$ was identified as a combined muon, and $(0.101 \pm 0.014) \%$ as a combined or segment tagged muon. The combined muon measurement is more redundant than the segment tagged one, and therefore has a higher background rejection.

In all studies, a good overall agreement is found between simulation and data, albeit with some detector regions where the data is significantly worse than the simulation, possibly due to chamber misalignment.

\section{References}

[1] ATLAS Collaboration, G. Aad et al., "The ATLAS Experiment at the CERN Large Hadron Collider", JINST 3 (2008) S08003, http://dx.doi.org/10.1088/1748-0221/3/08/S08003.

[2] ATLAS Collaboration, G. Aad et al., "Commissioning of the ATLAS Muon Spectrometer with cosmic rays", EPJ-C, 2010, http://dx.doi.org/10.1140/epjc/s10052-010-1415-2.

[3] ATLAS Collaboration, "Muon Reconstruction Performance", ATLAS note, ATLAS-CONF-2010-064, CERN, Geneva, Switzerland, 2010, http://cdsweb.cern.ch/record/1281339.

[4] ATLAS Collaboration, "Extraction of the prompt muon component in inclusive muons produced at $\sqrt{ } s=7 T e V^{\prime}$, ATLAS note, ATLAS-CONF-2010-075, CERN, Geneva, Switzerland, 2010, http://cdsweb.cern.ch/record/1281366. 